\title{
Une espèce nouvelle de Trichoptère du Portugal : Tinodes aravil n.sp. (Trichoptera : Psychomyiidae)
}

\author{
L.S.W. Terral \\ M.A. González²
}

Mots clés : Trichoptera, Psychomyiidae, espèce nouvelle, Portugal.

L'imago or de Tinodes aravil, espèce nouvelle du Portugal, est décrite. Elle est isolée dans le cadre du genre par la forme des gonopodes de même que par la conformation du complexe phallique.

A new species of caddisfly from Portugal : Tinodes aravil n.sp. (Trichoptera : Psychomyidae)

Keywords : Trichoptera, Psychomyiidae, new species, Portugal.

The male imago of Tinodes aravil, a new species from Portugal is described. The shape of the claspers and the structure of the phallic apparatus provide a striking distinction between this species and all others described previously in the genus. Close relationships to any other species are not evident.

Parmi les Trichoptères récoltés récemment par l'un de nous ( $L$. Terra) au cours de nos recherches sur les cours d'eau du centre du Portugal (Beira Baixa), nous avons découvert une espèce nouvelle du genre Tinodes, portant ainsi à 12 le nombre des espèces de ce genre connues de la Péninsule ibérique (González et al. 1992). On trouvera ci-après la description de cette nouvelle espèce.

\section{Tinodes aravil n. sp.}

\section{Matériel}

Terre typique : le Portugal ; Holotype mâle et 13 paratypes mâles du Portugal, Pardinhos (Monforte da Beira, Beira Baixa), Ribeira de Aravil, $170 \mathrm{~m}$, 3-X-91 (leg. L. Terra). 1 paratype mâle de ribeira do Alvito (Beira Baixa), $180 \mathrm{~m}, 1-\mathrm{X}-91$ (leg. L. Terra).

L'holotype et 7 paratypes ont été déposés dans la collection du Departamento de Biología Animal (Universidad de Santiago) ; 7 paratypes sont

1. Estaçao Aquicola, 4481 Vila do Conde codex, Portugal,

2. Departamento de Biologia Animal, Facultad de Biologia, Universidad de Santiago, 15706 Santiago de Compostela, España. conservés dans la collection de la Estaçao Aquicola (Vila do Conde). Tous les exemplaires sont conservés dans l'alcool.

\section{Description de l'imago mâle}

Coloration générale du corps variant de jaune clair à brunâtre. Dessus de la tête et du thorax et palpes maxillaires brunâtres. Antennes jaunes mais nettement annelées de brun au niveau des articulations . Pattes blanchâtres. Ailes jaune clair. Longueur de l'aile antérieure : $6,0-7,3 \mathrm{~mm}$.

Genitalia (Fig. 1-7) :

Xème segment apparaissant triangulaire, en vue de profil (Fig. 1), aux contours irréguliers, avec bord antérieur nettement concave et bords ventral et postérieur sinueux, ceux-ci formant un lobe obtus à l'angle ventro-distal. En vue ventrale (Fig. 2), le Xème segment forme une plaque à peu près pentagonale, avec bord antérieur arrondi et bord postérieur légèrement découpé.

Appendices supérieurs longs, atteignant l'extrémité des gonopodes, minces mais légèrement élargis à l'apex et nettement recourbés vers le bas (Fig. 1). 

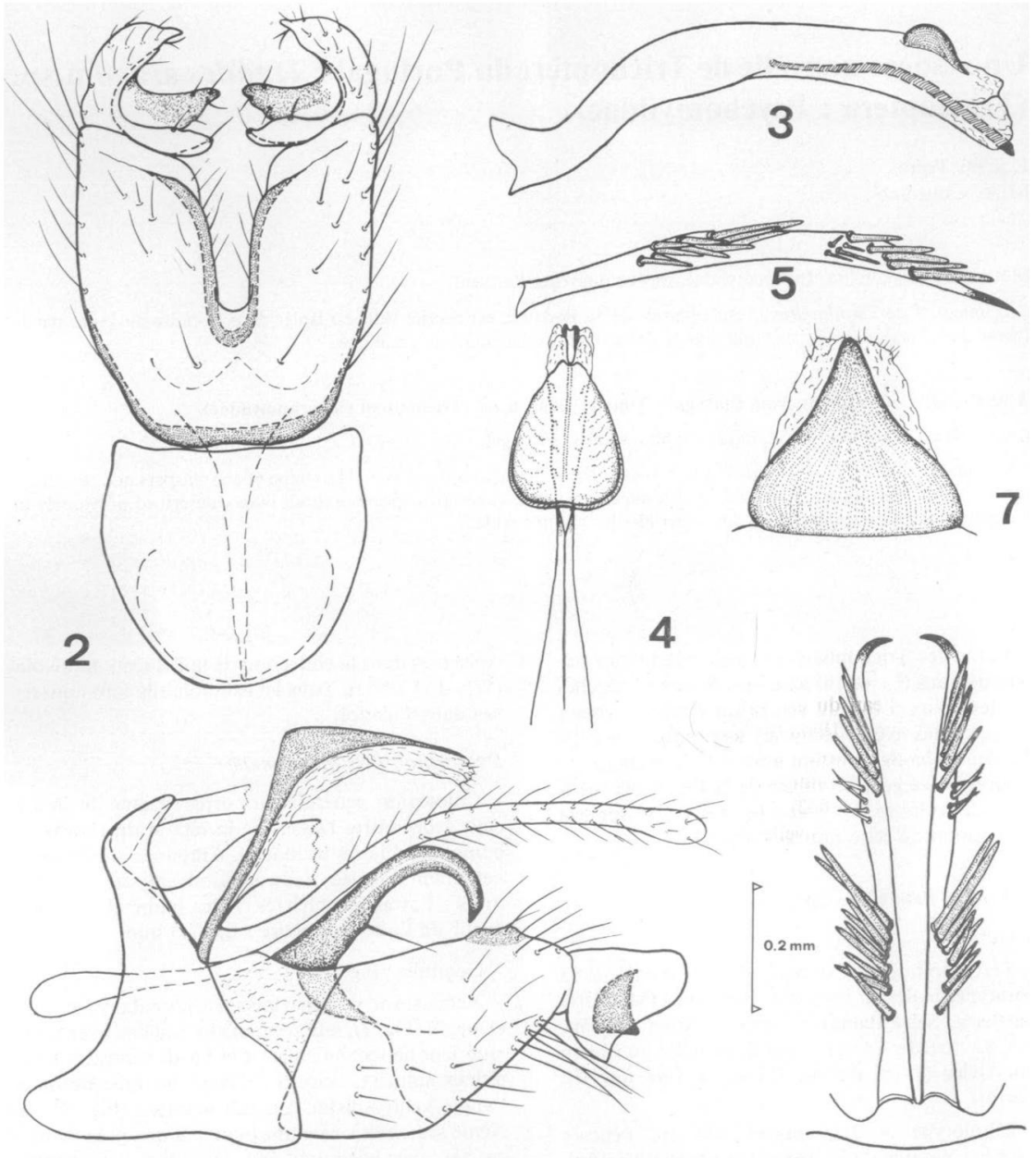

1

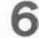

Fig. 1-7. Tinodes aravil n. sp. 1. genitalia mâie, vue latérale (seule la racine du phallus et du paramère est représentée); 2 . vue ventrale du IXème segment et des gonopodes; 3. phallus, vue latérale ; 4. phallus, partie distale, vue dorsale (dans ce cas échelle graphique équivalente à $0,15 \mathrm{~mm}) ; 5$. paramère, vue latérale ; 6 . paramères, vue dorsale; 7 . IXème tergite, vue dorsale.

Fig. 1-7. Tinodes arovil n. sp. 1. male genitalia lateral view (only the root of the phallus and paramere is represented); 2 . ventral view of IX segment and claspers ; 3 . phallus, lateral view ; 4. phallus, distal part, dorsal view (graphic scale equivalent to $0.15 \mathrm{~mm}$ ) ; 5. paramere, lateral view ; 6. parameres, dorsal view ; 7 . IX tergite, dorsal view. 
Les appendices inférieurs permettent de distinguer facilement le coxopodite, l'harpago et l'armature interne (médiane). L'aspect latéral des coxopodites (Fig. 1) est bien caractéristique, en forme de rhomboïde, portant une petite callosité près de l'angle distal-supérieur (parfois peu distincte) ; le contour de l'ensemble est, en vue ventrale (Fig. 2), rectangulaire sur sa moitié distale, puis se rétrécit nettement vers sa partie basale. Harpago fort bien dégagé, nettement trilobé en vue ventrale (Fig. 2) : on y distingue un lobe supérieur, un lobe inférieur et un lobe médian. Les deux lobes supérieur et inférieur sont séparés par un sillon très profond ; le lobe supérieur est dirigé obliquement vers le haut, en forme de crochet, avec l'apex brusquement aminci et dirigé vers l'intérieur, tandis que le lobe inférieur est plus gracile et à peu près horizontal. Le lobe médian, situé entre les deux précédents, est en forme de triangle étiré, disposé horizontalement ; leur partie basale, fort peu sclérifiée fait saillie de la concavité de la face interne de l'harpago. L'aspect de l'harpago en vue latérale (Fig. 1) est assez différent : il forme une plaque robuste subtriangulaire, obtusément arrondie à l'apex et avec son angle basal inférieur portant une ailette triangulaire recourbée vers l'intérieur. La plaque basale-médiane des gonopodes présente un apodème assez court, aplati sagittalement, qui est très légèrement recourbé vers le haut en vue latérale (Fig. 1) et assez net tement élargi à l'extrémité céphalique ; la branche dorsale (processus de la plaque basale) est, en vue latérale (Fig. 1), un peu élargie à son tiers basal, puis régulièrement rétrécie vers l'apex qui est très pointu et très nettement recourbé vers le bas.

Le complexe phallique est composé d'un phallus et des paramères (processus paraproctaux). Ceuxci ont une conformation assez caractéristique ; en vue dorsale (Fig. 6) ils apparaissent grêles et légèrement divergents, avec un apex en pointe acérée et visiblement recourbé vers la ligne médiane. Vus de profil (Fig. 5), les paramères apparaissent légèrement et régulièrement recourbés vers le bas. Sur ses faces dorsale et latérale s'insèrent deux groupes d'épines : un groupe basal composé de 8-12 épines, fort serrées, de taille inégale, dont les plus longues sont celles de l'extrémité distale et un autre, à la partie préapicale composé de 4-6 épines longues et de 4 petites spinules. Phallus assez fortement sclérifié, sauf à l'extrémité apicale qui reste membraneuse ; aedeagus visible par transparence. Vu de profil (Fig. 3), le phallus est aminci dans sa région basale (qui est oblique vers le haut), puis de largeur à peu près régulière sur le reste qui est légèrement arqué vers le bas. Le phallus se trouve assez fortement aplati latéralement à sa moitié distale ; en vue de dessus (Fig. 4), dans cette région, particulièrement au niveau de la partic basale du lobe dorsal, il apparaît fortement rétréci ; derrière ce lobe se trouve une longue carène dorsale médiane. Le bord supérieur de la partie préapicale du phallus porte, de chaque côté, une sorte d'ailette latérale arrondie, dressée obliquement vers l'extérieur ; vues de dessus, les deux ailettes apparaissent fusionnées l'une à l'autre et dèlimitent une sorte de lobe en forme de cceur.

\section{Affinités}

Il est hasardeux de faire actuellement des considérations sur les affinités de cette nouvelle espèce. Elle nous semble assez isolée, très bien individualisée dans les limites du genre par la forme des gonopodes - particulièrement l'aspect nettement trilobé de l'harpago en vue ventrale - et du complexe phallique.

\section{Travaux cités}

González M.A., Terra L.S.W., Garcia de Jalón D. \& Cobo F. 1992. - Lista faunística y bibliográfica de los Tricópteros (Trichoptera) de la Peninsula Iberica e Islas Baleares. Assoc. esp. Limnol. (Ed.), Publ. $n^{\circ} 11: 200$ p. 